\title{
Relato de um caso de transmissão transplacentária de anaplasmose bovina. Observações clínico-laboratoriais
}

\section{Occurence of transplacental transmission of bovine anaplasmosis. Clinical and laboratorial observations}

\author{
Fernando José Benesi, ${ }^{*}$ Denise Louise Howard, ${ }^{\star \star}$ Cristiano dos S. Cardoso de Sá, ${ }^{\star \star \star}$ Eduardo Harry Birgel Junior ${ }^{\star}$
}

\begin{abstract}
Resumo
Relata-se a ocorrência de transmissão transplacentária de anaplasmose em um bezerro da raça Jersey, com 7 dias de vida, proveniente de propriedade localizada no Município de São Miguel Arcanjo, Estado de São Paulo, destacando-se as observações clínico-laboratoriais realizadas durante a evolução do quadro nosológico.
\end{abstract}

Palavras-chave: anaplasmose; transmissão transplacentária; Anaplasma marginale.

A anaplasmose é uma enfermidade infecciosa caracterizada por apatia, febre, anemia e icterícia, sendo os carrapatos da espécie Boophilus microplus considerados os principais vetores da ricketsia causadora desta enfermidade. Vários relatos da ocorrência da anaplasmose em bezerros neonatos, demonstrando a possibilidade da transmissão transplacentária do Anaplasma marginale (Bird, 1973; Payne, Miller, 1977), têm comprovado a importância desta via de transmissão na determinação da hemoparasitose nesses recém-nascidos na primeira semana de vida (Zaugg, 1985 ; Potgieter, Rensburg, 1987 ; Salabarria, Pino, 1988), sendo a infecção fetal evidenciada em $2,1 \%$ dos animais nascidos em regiões endêmicas, e em $36,0 \%$ daqueles filhos de fêmeas premunidas durante a gestação (Ribeiro et al., 1995). Os trabalhos de Zaugg (1985), Salabarria, Pino (1998) e de Ribeiro et al. (1995) também destacaram que a infecção fetal por esta via ocorria particularmente no $3^{\circ}$ trimestre de gestação.

No Brasil, os relatos da ocorrência da anaplasmose, com as manifestações clínicas da enfermidade observadas na primeira semana de vida, são raros (Passos, Lima, 1984; Klobucaric et al., 1986), motivando a presente comunicação sobre as observações clínico-laboratoriais da anaplasmose realizadas durante a evolução da anaplasmose neonatal.

Em maio de 1997 foi encaminhado ao Serviço de Clínica Médica de Grandes Animais do Departamento de Clínica Médica-VCM/ Hospital Veterinário-HOVET, da Faculdade de Medicina Veterinária e Zootecnia da Universidade de São Paulo, um bezerro da raça Jersey, macho, com 7 dias de vida, proveniente de propriedade localizada no Município de São Miguel Arcanjo, Estado de São Paulo.
Ao exame físico inicial, constatou-se ligeira apatia, decúbito esternal, mufla com umidade diminuída e fezes firmes. Em relação às funções vitais, observou-se hipertermia (temperatura retal $-39,9^{\circ} \mathrm{C}$ ), estando as freqüências cardíaca (96 batimentos por minuto) e respiratória (36 movimentos por minuto) dentro dos limites fisiológicos. As mucosas oculares apresentavam coloração rosa-pálida e tonalidade levemente amarelada. Evidenciou-se, ainda, respiração rude com ligeira propagação dos batimentos cardíacos em área pulmonar.

O exame do eritrograma (Tabela 1), realizado segundo as técnicas descritas por Birgel (1982), revelou a presença de anemia (Hemácias - 2,8 $\times 10 \% / \mathrm{mm}^{3}$; Volume Globular - $12 \%$; Taxa de Hemoglobina - 5,3g/dl) do tipo normocítica (VCM $33,4 \mathrm{~m}^{3}$ ) , eritrorregenerativa (com presença de eritroblastos e policromasia moderada) e que evoluiu tornando-se macrocítica e normocrômica (VCM - 67,2m³ e CHCM - 31,3\%), constatando-se a presença de corpúsculos típicos de Anaplasma marginale em $20 \%$ das hemácias observadas.

O perfil bioquímico (Tabela 1), avaliado segundo as técnicas descritas por Souza (1996), revelou como principal alteração a presença de hiperbilirrubinemia indireta, com maior magnitude no $3^{\circ}$ dia de observação (Bilirrubinas Total - 4,36mg/dl; Indireta - 3,99mg/dl; e Direta - 0,37mg/dl). O animal recebeu tratamento durante dois dias através da aplicação de tetraciclina em dose de $11 \mathrm{mg} / \mathrm{kg} \mathrm{p.v./dia.} \mathrm{No} \mathrm{decorrer} \mathrm{do} \mathrm{período} \mathrm{de} \mathrm{observa-}$ ção, verificou-se ausência da síndrome, febre a partir do segundo dia, com normalização da coloração de mucosas e dos componentes do eritrograma no sétimo dia de avaliação.

O quadro sintomático observado (apatia, febre, anemia e icterícia) permitiu caracterizar-se a ocorrência da hemoparasitose

\footnotetext{
^Docentes do Departamento de Clínica Médica, Faculdade de Medicina Veterinária e Zootecnia, Universidade de São Paulo.

**Mestranda Capes.

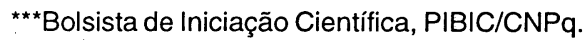


em neonato, com manifestações similares às descritas por Bird (1973), Payne, Miller (1977), Passos, Lima (1984), Klobucaric et al. (1986), sendo a confirmação do diagnóstico etiológico efetuada pela comprovação da presença do

Tabela 1: Observações clínico-laboratoriais realizadas em bezerro recém-nascido infectado por Anaplasma marginale

\begin{tabular}{|c|c|c|c|c|c|c|c|}
\hline Observacao i dia & 10 & $2^{3}$ & $3^{\prime}$ & $4^{2}$ & $5^{\prime \prime}$ & $6^{2}$ & $7^{2}$ \\
\hline Tratamento* & + & + & - & - & - & - & - \\
\hline Temperatura retal ${ }^{* *}$ & $\uparrow$ & $N$ & $\mathrm{~N}$ & $\mathrm{~N}$ & $N$ & $\mathrm{~N}$ & $N$ \\
\hline $\mathrm{He}\left(\times 10^{6} / \mathrm{mm}^{3}\right)^{* * *}$ & 2,8 & 3,4 & 3,2 & 3,5 & 2,7 & 3,6 & 5,1 \\
\hline $\mathrm{Ht}(\%)$ & 12 & 20 & 20 & 18 & 18 & 22 & 26 \\
\hline $\mathrm{Hb}(g / d)$ & 5,3 & 5,8 & 6,4 & 6,4 & 5,6 & 6,2 & 8,4 \\
\hline $\operatorname{VCM}\left(\mu^{3}\right)$ & 33,4 & 59,3 & 62,5 & 51,4 & 67,2 & 61,1 & 51,0 \\
\hline $\mathrm{CHCM}(\%)$ & 44,1 & 29,0 & 31,8 & 35,4 & 31,3 & 28,2 & 32,3 \\
\hline \multicolumn{8}{|l|}{ Outras obsenaçōes } \\
\hline Eritrorregeneração **** & $+(+)^{-}$ & $+(+)-$ & $+(+)-$ & $+^{-}$ & $H^{-}$ & $+\cdots$ & $+\cdots$ \\
\hline Eritroblastos/100 Le***** & 5 & 1 & 0 & 1 & 5 & 0 & 0 \\
\hline Hemoparasitas (Anaplasma sp) - \% & 20,0 & 14,0 & 1,0 & 5,0 & 2,5 & 2,5 & 0 \\
\hline Proteina Total $(g / d l)$ & 4.6 & 5.3 & 5.3 & 5.1 & 5.1 & 5.5 & 5.9 \\
\hline Albumina $(g / d l)$ & 2.6 & 2.9 & 2.9 & 2.7 & 3.2 & 3.1 & 3.2 \\
\hline AST (U/I) & 10.0 & 12.0 & 12.0 & 11.0 & 15.0 & 19.0 & 17.0 \\
\hline GGT (U/I) & 10.0 & 10.0 & 11.0 & 13.0 & 11.0 & 12.0 & 11.0 \\
\hline Uréia (mg/dl) & 27.0 & 23.0 & 15.0 & 13.0 & 18.0 & 26.0 & 21.0 \\
\hline Creatinina $(\mathrm{mg} / \mathrm{dl})$ & 0.91 & 0.86 & 0.92 & 1.07 & 1.05 & 0.96 & $1: 13$ \\
\hline Bilimubina Total (mg/dl) & 2,96 & 3,91 & 4,36 & 2,85 & 4,11 & 3,66 & 1,56 \\
\hline Bilimubina Direta (mg/dl) & 0,22 & 0,23 & 0,37 & 0,10 & 0,25 & 0,29 & 0,15 \\
\hline Bilimubina Indireta(mg/dl) & 2,74 & 3,68 & 3,99 & 2,75 & 3,86 & 3,37 & 1,41 \\
\hline
\end{tabular}

* $+=$ realizado - = não realizado; ${ }^{\star \star}+=$ acima do normal/ $\mathrm{N}=$ normal; ${ }^{* \star \star} \mathrm{He}=$ número de hemácias; $\mathrm{Ht}=$ volume globular; $\mathrm{Hb}=$ taxa de hemoglobina; $\mathrm{VCM}=$ Volume Corpuscular Médio; $\mathrm{CHCM}=$ Concentração Hemoglobínica Corpuscular Média; ${ }^{\star \star \star \star \star}$ $+++=$ intensa/ $++-=$ moderada/ $+(+)$ - leve a moderada/ $+-=$ leve; ${ }^{\star \star \star \star \star}$ Le $=$ Leucócitos contados.
Anaplasma marginale em hemácias, através da avaliação do esfregaço sangüíneo corado, conforme relatado em outras pesquisas (Bird, 1973; Payne, Miller, 1977; Passos, Lima, 1984; Klobucaric et al.,1986). Os sinais de comprometimento do Sistema Nervoso Central, com opistótono e nistagmo, descritos porBird (1973) e Klobucaric et al. (1986), não foram assinalados no animal da presente comunicação. Os resultados obtidos na avaliação laboratorial complementar permitiram a caracterização morfotintorial e o caráter regenerativo da anemia, além do momento da recuperação hematológica do neonato, fatos não relatados na literatura (Bird,1973; Klobucaric et al., 1986). Nestas últimas pesquisas a icterícia foi caracterizada como sendo pré-hepática e causada por crise hemolítica, comprovada pela hiperbilirrubinemia indireta. Todavia, no presente relato, além deste fato, pode-se relacionar a normalização da cor das mucosas aparentes com a queda dos níveis séricos das bilirrubinas.

A correlação entre a idade do bezerro afetado e o longo período de incubação da anaplasmose bovina permitiu concluir-se que - animal sofreu infecção pelo Anaplasma marginale, durante o período fetal, através de transmissão transplacentária, confirmando-se as evidências das pesquisas e relatos de casos similares colhidos na literatura pertinente ao assunto (Zaugg, 1985 ; Salabarria, Pino, 1988 ; Potgieter, Rensburg, 1987 ; Ribeiro et al., 1995).

\section{Abstract}

This study relates the occurence of transplacental transmission of anaplasmosis to a seven-day Jersey calf, provenient from a propriety in São Miguel do Arcanjo, State of São Paulo, with emphasis in the clinical and laboratorial observations made during the evolution of the case.

Keywords: anaplasmosis; transplacental transmission; Anaplasma marginale.

\section{Referências bibliográficas}

BIRD, J. E. Neonatal anaplasmosis in a calf. J. S. Afr. Vet. Assoc., n. 44, p. 69-70, 1973.

BIRGEL, E. H. Hematologia Clínica Veterinária. In: BIRGEL, E. H., BENESI, F. J. Patologia Clínica Veterinária. São Paulo: Sociedade Paulista de Medicina Veterinária, p. 2-50, 1982.

KLOBUCARIC, A., BENESI, F. J., BIRGEL, E. H. Ocorrência de anaplasmose em bezerro recém-nascido. In: SEMANA DE VETERINÁRIA, 4., 1986, São Paulo. Anais, São Paulo : Faculdade de Medicina Veterinária e Zootecnia da Universidade de São Paulo, 1986, p. 12.

PASSOS, L. M. F. LIMA, J. D. Diagnóstico de anaplasmose bovina congênita em Minas Gerais. Arq. Bras. Med. Vet. Zoot. n. 36, p. 743- 744, 1984.

PAYNE, G. D., MILLER, A. S. Anaplasmosis in a newborn calf. Vet. Rec. n. 100, p. 58, 1977.

POTGIETER, F.T., VAN RENSBURG, L. The persistence of colostral anaplasma antibodies and incidence of in utero transmission of anaplasma infections in calves under laboratory conditions. Onderstepoort J. Vet. Res., n. 54, p. 557-560, 1987.

RIBEIRO, M. F. B., LIMA, J. D., GUIMARÃES, A. M., SCATAMBURLO, M. A., MARTINS, N. E. Transmissão congênita da anaplasmose bovina. Arq. Bras. Med. Vet. Zootec. n. 47, p. 297-304, 1995.

SALABARRIA F. F., PINO, R. Transmission vertical de Anaplasma marginale en bovinos afetados durante el periodo final dela gestacion. Rev. Cub. Cienc. Vet, n. 19, p. 179-181, 1988.

SOUZA, P. M. 1997. Perfil bioquímico sérico de bovinos das raças gir, holandesa e girolanda, criados no Estado de São Paulo. Influência de fatores de variabilidade etários e sexuais. 1986. 168 p. Tese (Doutorado) - Faculdade de Medicina Veterinária e Zootecnia da Universidade de São Paulo, 1986.

ZAUGG, J. L. Bovine anaplasmosis: Transplacental transmission as it relatès to stage of gestation. Am. J. Vet. Res. n. 46, p. 570-572, 1985. 